\title{
Design and Performance of an Electrically Small Slot Loop Antenna With a Miniaturized Superconducting Matching Circuit
}

\author{
Y. Tsutsumi, H. Kanaya, and K. Yoshida
}

\begin{abstract}
In order to realize a small superconducting receiver, we designed an electrically small slot loop antenna with a miniaturized impedance matching circuit. The area of the slot loop antenna is $4 \mathrm{~mm} \times 4 \mathrm{~mm}$ on $\mathrm{MgO}$ substrate at a frequency of $2.4 \mathrm{GHz}$. By applying 2-pole bandpass filter technique, we propose a design theory of the miniaturized impedance matching circuit that consists of coplanar waveguide (CPW) quarter and half wavelength transmission lines of meandering geometry and the impedance inverting circuits ( $\mathrm{J}$ inverters). By using electromagnetic field simulator, we realized the circuit which matches the small radiation resistance $R_{a}=0.776 \Omega$ of the electrically small slot loop antenna to the feeder impedance $Z_{0}=50 \Omega$, and attained the normalized bandwidth of $3 \%$. We also made a preliminary experiment on this miniaturized antenna using a YBCO thin film on MgO substrate.
\end{abstract}

Index Terms-Coplanar waveguide, miniaturized impedance matching circuit, slot loop antenna.

\section{INTRODUCTION}

D URING recent years, miniaturized planar antennas are essential to radio communication devices such as wireless LAN, RF-ID and MIMO in information society, because the size of antenna often has a great influence on the whole size of a wireless system [1]-[5].

The characteristic of the antenna is closely concerned with its size. Some antennas are designed at the cost of their bandwidth for miniaturization [1]. On the other hand, many studies have done for wide bandwidth [2]. Antennas fed by CPW, that have the advantages such as low radiation loss, flexible configuration and so on, have also studied frequently [3]-[5].

There are also many reports on high-temperature superconducting (HTS) antennas, which have high efficiency due to high $\mathrm{Q}$ of superconducting resonators [6]-[11]. A small antenna is sensitive to the conductor resistance because its radiation resistance is small. Thus, an HTS antenna, whose electrode resistance is negligibly small, is useful for miniaturization.

Manuscript received October 5, 2004. This work was supported in part by a Grant-in-Aid for Scientific Research (B) from the Japan Society for the Promotion of Science (JSPS) and in part by a grant of Fukuoka project in the Cooperative Link of Unique Science and Technology for Economy Revitalization (CLUSTER) of the Ministry of Education, Culture, Sports, Science and Technology (MEXT).

The authors are with the Department of Electronics, Graduate School of Information Science and Electrical Engineering, Kyushu University, Fukuoka 8128581 (e-mail: yoshida@ed.kyushu-u.ac.jp).

Digital Object Identifier 10.1109/TASC.2005.850187

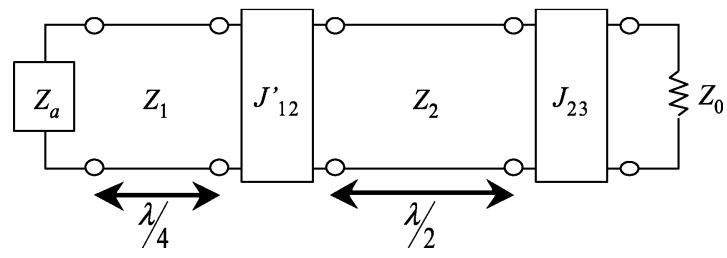

Fig. 1. The equivalent circuit of the matching circuit for the antenna.

In our previous works, we have designed slot dipole antenna whose length is one wavelength [8] and the antenna connected to the low noise amplifier with a prototype matching circuit using CPW [9], [10]. In order to increase the directivity of the antenna, array antenna was also designed by folding the slot antenna and branching out the feed line [10], [11].

In this paper, we propose a new design formula of an electrically small antenna (ESA), that is, an antenna whose dimension is much smaller than one wavelength, with a miniaturized matching circuit. In the present study, we designed a slot loop antenna with a 2-pole matching circuit using CPW. Furthermore, we tested its performance by experiment as well as electromagnetic field simulator.

\section{Design of IMPEDANCE MATChING CiRCUIT FOR ESA}

Fig. 1 shows the circuit model of the proposed matching circuit for the antenna, which was obtained by modifying the design theory given in [8], [12]. The matching circuit becomes smaller by using a quarter wavelength transmission line. In the figure, $Z_{a}=R_{a}+\mathrm{j} X_{a}$ and $Z_{0}(=50 \Omega)$ are the impedance of the antenna and the external port, respectively. The proposed design values for the characteristic impedance of the transmission line $\left(Z_{1}\right)$ and J-parameters $\left(J_{12}^{\prime}, J_{23}\right)$ are given by

$$
\begin{aligned}
Z_{1} & =\frac{4}{\pi} R_{a}\left(Q_{e 1}-Q_{a}\right) \\
J_{12}^{\prime} & =w \sqrt{\frac{b_{1}^{\prime} b_{2}}{g_{1} g_{2}}} \\
J_{23} & =\sqrt{w} \sqrt{\frac{b_{2}}{Z_{0} g_{2} g_{3}}}
\end{aligned}
$$




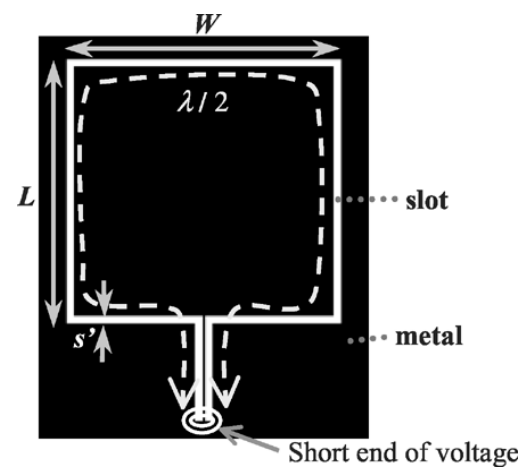

Fig. 2. Configuration of the slot loop antenna.

with

$$
\begin{aligned}
b_{1} & =\frac{\pi}{4} \frac{1}{Z_{1}} \\
b_{1}^{\prime} & =\frac{b_{1}}{1-\frac{Q_{a}}{Q_{e 1}}} \\
b_{2} & =\frac{\pi}{2} \frac{1}{Z_{2}} \\
Q_{a} & =\frac{x_{a}}{R_{a}} \\
Q_{e} & =\frac{g_{0} g_{1}}{w} \\
x_{a} & =\left.\frac{\omega_{0}}{2} \frac{\partial X_{a}}{\partial \omega}\right|_{\omega=\omega_{0}},
\end{aligned}
$$

where $x_{a}$ is the reactance slope parameter at the series resonance of the antenna impedance [8], $w$ is the normalized frequency bandwidth, and $g_{i}(i=0,1,2,3)$ is the normalized filter element, $Q_{a}$ and $Q_{e}$ are the unloaded $\mathrm{Q}$ of antenna and the external $\mathrm{Q}$, respectively, and $b_{i}(i=1,2)$ is the susceptance slope parameter.

It is noted that, because the present design theory of the matching circuit is based on that of the bandpass filter [12], it is possible not only to match impedance but also to adjust the bandwidth $w$.

\section{SimUlation OF SLOT LOOP ANTENNA}

\section{A. Slot Loop Antenna}

The structure of proposed slot loop antenna is shown Fig. 2. The total length of the slot loop including feed line is half of effective wavelength. The backend of the loop is the voltage short end, thereby the whole operates as an in-series resonator.

As shown in Fig. 3, the radiation resistance $\left(R_{a}\right)$ and the reactance slope parameter $\left(x_{a}\right)$ of the slot loop antenna are dependent on both the area of the loop and the slot width at $2.45 \mathrm{GHz}$. In order to widen the bandwidth of the antenna, that is, to lower the $\mathrm{Q}$ value of the antenna $\left(Q_{a}\right)$, the area of the loop portion needs to be expanded. In order to miniaturize of the antenna, the value of $L(=W)$ is set as $4000 \mu \mathrm{m}$. The frequency dependence of the input impedance of the slot loop antenna $\left(Z_{a}\right)$ is shown in Fig. 4. Thereby, $R_{a}=0.776 \Omega$ and $x_{a}=13.9 \Omega$ are obtained for the gap spacing $s^{\prime}=15.5 \mu \mathrm{m}$.
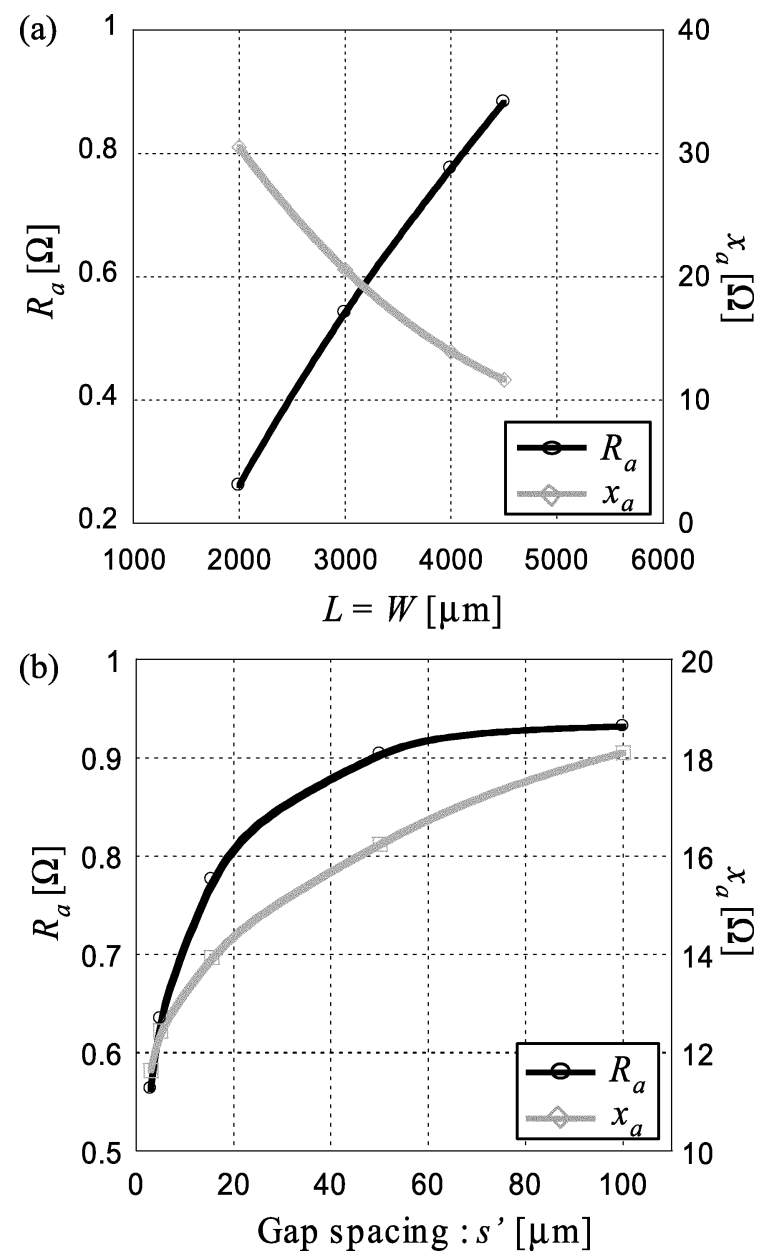

Fig. 3. (a) $L$ and $W$ (parameter: $s^{\prime}=15.5 \mu \mathrm{m}$ ) dependence of $R_{a}$ and $x_{a}$, (b) $s^{\prime}$ (parameters: $L=W=4000 \mu \mathrm{m}$ ) dependence of $R_{a}$ and $x_{a}$.

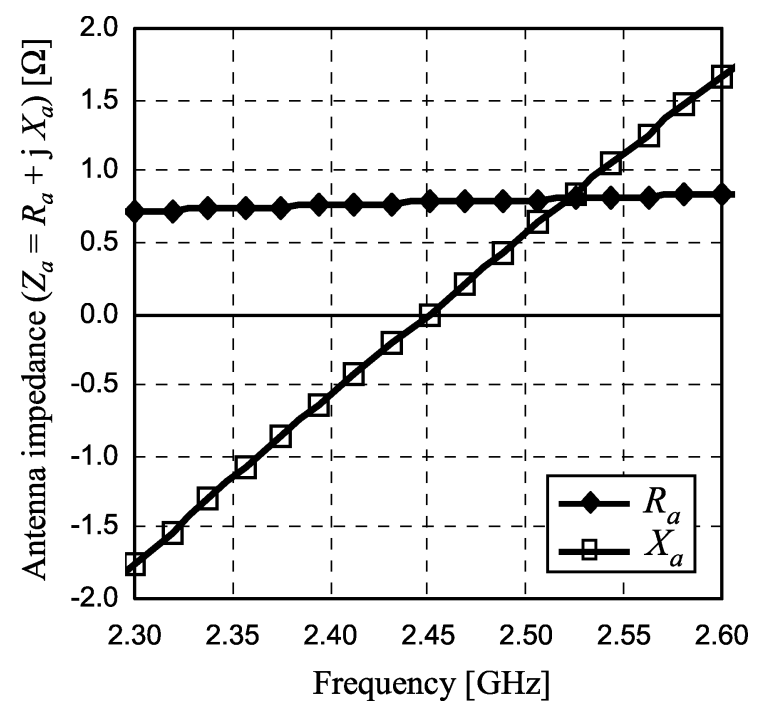

Fig. 4. Antenna impedance of the slot loop antenna. Parameters: $L=W=$ $4000 \mu \mathrm{m}, s^{\prime}=15.5 \mu \mathrm{m}$.

\section{B. Slot Loop Antenna With Matching Circuit Using CPW}

Fig. 5 shows the layout of the slot loop antenna with 2-pole matching circuit. $\mathbf{J}$ inverters consist of interdigital gap and in-series resonators consist of meandering coplanar waveguide. Fig. 6 shows the frequency dependence of the return loss. 


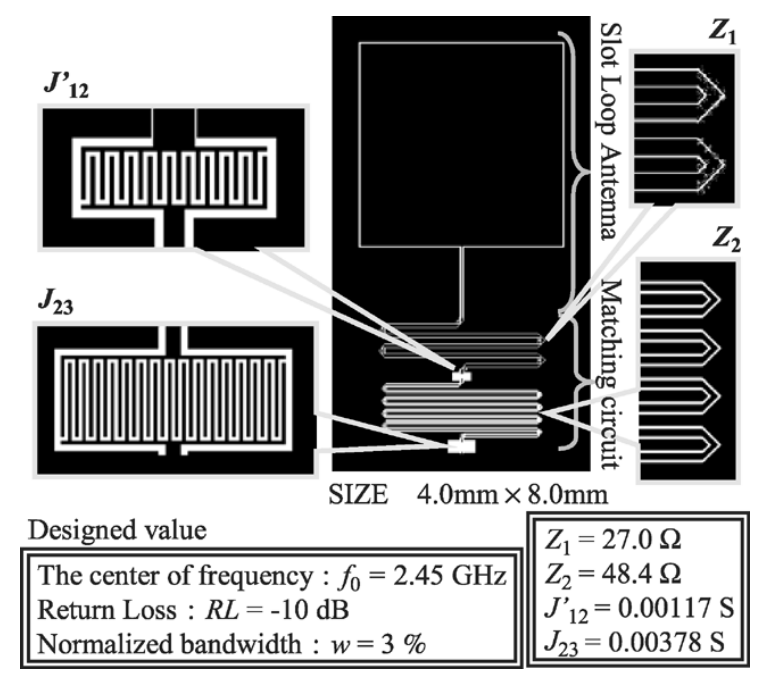

Fig. 5. The layout and design value of the slot loop antenna with 2-pole matching circuit.

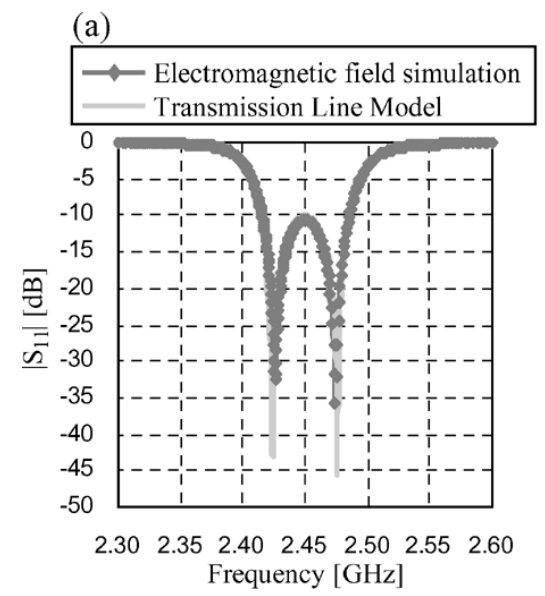

(b)

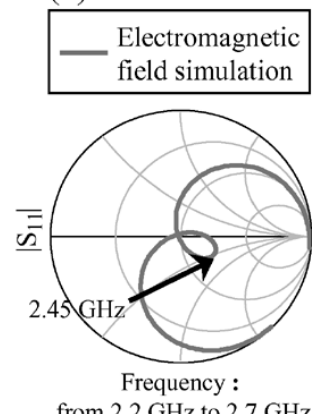

Fig. 6. The frequency dependence of $\left|\mathrm{S}_{11}\right|$ of the slot loop antenna with 2-pole matching circuit ((a) return loss, (b) Smith chart).

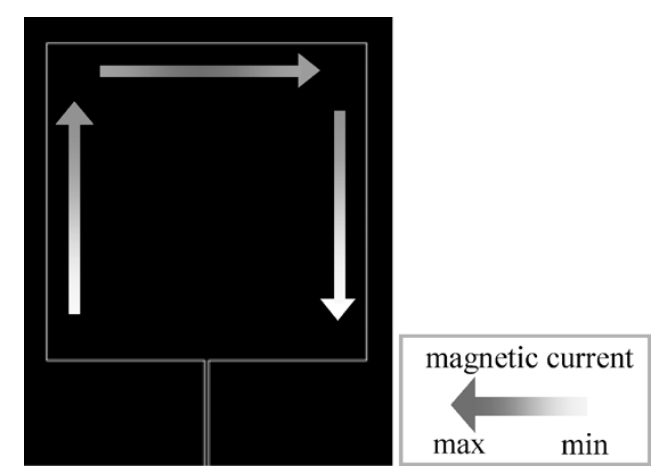

Fig. 7. The magnetic current of the slot loop antenna.

Simulation result is in fair agreement with the result of transmission line model. The desirable bandwidth $(R L=-10 \mathrm{~dB}$, $w=3 \%$ ) and the center of frequency $f_{0}=2.45 \mathrm{GHz}$ have been obtained, and impedance matching between $R_{a}=0.776 \Omega$ and $Z_{0}=50 \Omega$ is realized in the passband.

The magnetic current and radiation pattern are shown in Fig. 7 and Fig. 8, respectively. It is thought that because the magnetic current at the backend of a loop portion mainly radiates, the directivity pattern resembles that of dipole antenna.

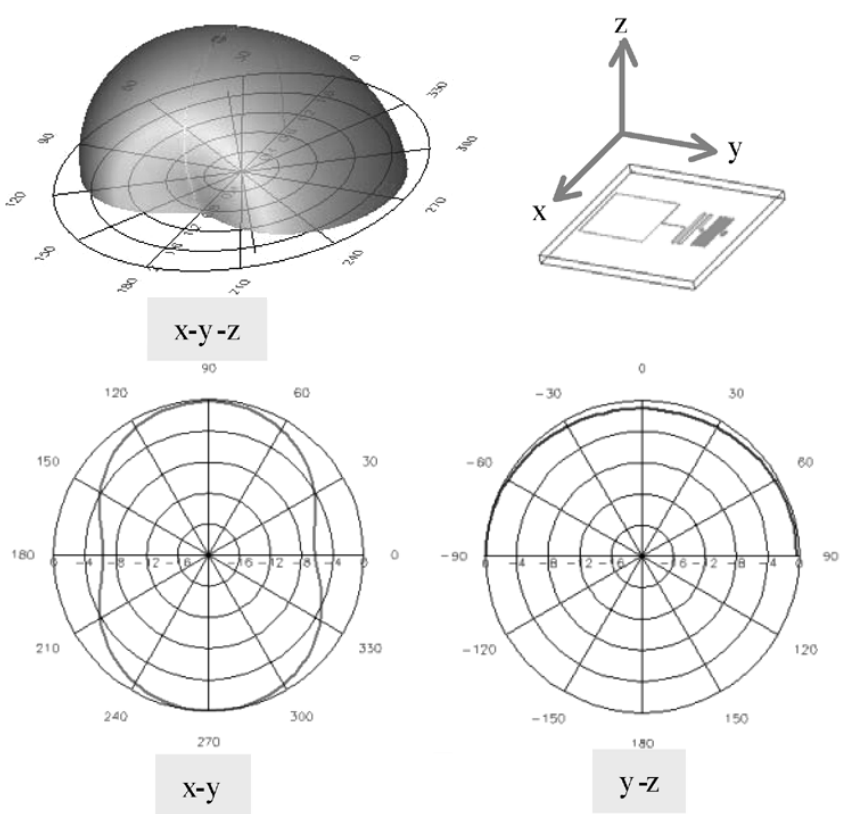

Fig. 8. Directivity pattern of the slot loop antenna.

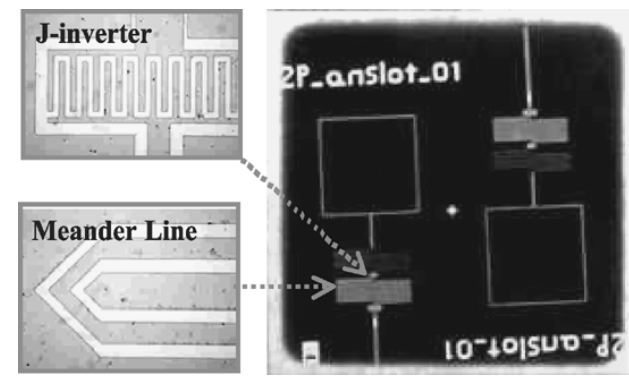

Fig. 9. Photograph of the slot loop antenna with 2-pole matching circuit. Substrate: $\mathrm{MgO}$ (size $15 \mathrm{~mm} \times 15 \mathrm{~mm}$ ), film: $\mathrm{YBa}_{2} \mathrm{Cu}_{3} \mathrm{O}_{7-x}$.

\section{EXPERIMENTAL RESULT}

Fig. 9 shows the photograph of the prototype YBCO slot loop antenna with the miniaturized matching circuit fabricated by the wet etching process. This antenna was placed in a vacuum cryostat, and was measured by using coplanar waveguide probes.

Figs. 10(a) and (b) show the experimental results for the return loss of the present miniaturized antenna. The frequency response of $S$-parameter was measured with vector network analyzer (HP-8722C) at $20 \mathrm{~K}$. In the figures, the results of transmission line models are also plotted for discussion. As for the return loss, the value of $-3 \mathrm{~dB}$ or less was obtained in the passband. Because of the over-etching and the residual loss from the connection of the probe, the return loss is inferior to those of the simulation results. It seems that the shift of the observed center frequency results from the influence of the kinetic inductance and/or the variation of $\mathrm{MgO}$ relative permittivity. As shown in Fig. 10(a), the center frequency that takes account of the kinetic inductance was shifted to the lower frequency. Similarly, as shown in Fig. 10(b) the center frequency simulated using larger dielectric constant value was shifted to the lower frequency too. These influences were obtained by changing the inductance and capacitance of transmission lines as a primary approximation in transmission line models. 

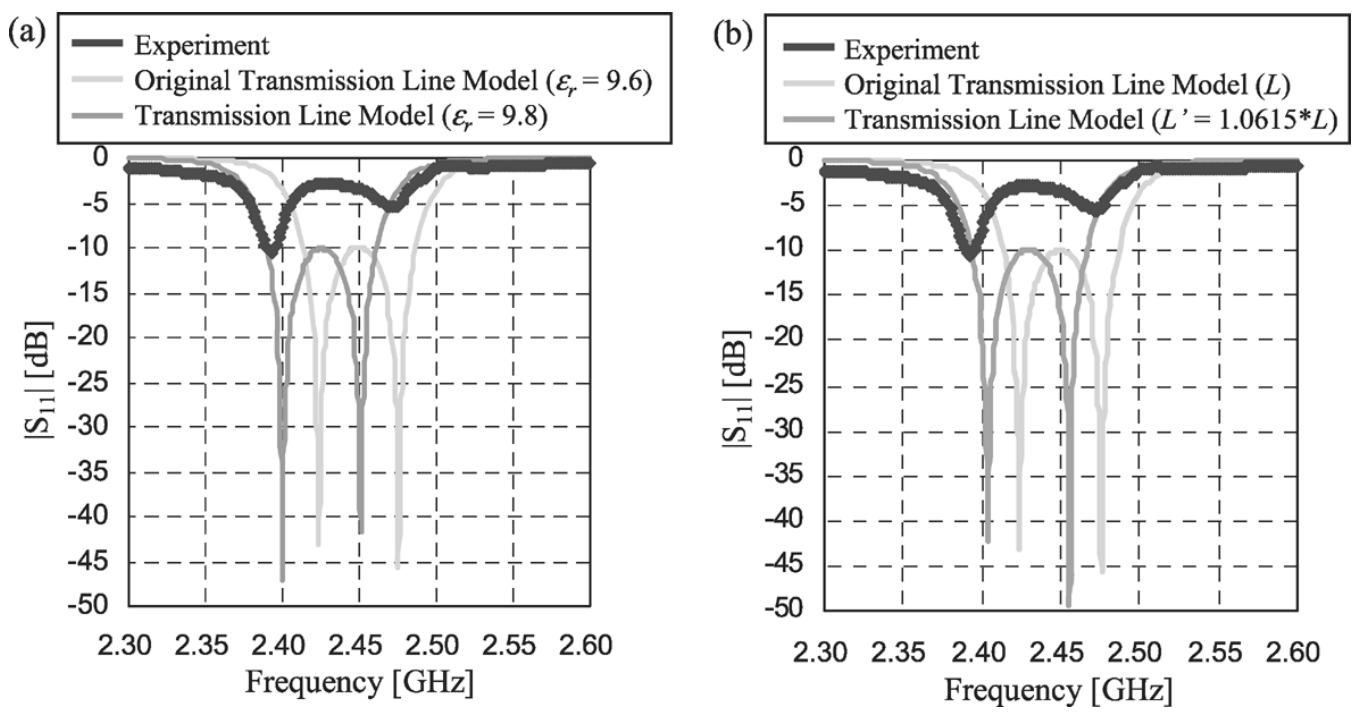

Fig. 10. Obtained frequency characteristic of the return loss and the calculations taking account of (a) kinetic inductance, and (b) permittivity variation.

However, the observed fractional bandwidth and the two peaks which are characteristic of $n=2$ filter are in reasonable agreement to the result of simulation.

\section{CONCLUSION}

In this paper, a slot loop antenna with a novel matching circuit based on 2-pole bandpass filter has been designed and tested. We succeeded to realize the circuit which matches the small radiation resistance $R_{a}(=0.776 \Omega)$ of ESA to the feeder impedance $Z_{0}(=50 \Omega)$. As a result, we designed a small $(4 \mathrm{~mm} \times 8 \mathrm{~mm})$ and broadband $(R L=-10 \mathrm{~dB}, w=3 \%)$ planar antenna. Moreover, YBCO slot loop antenna with 2-pole matching circuit was fabricated and tested in the cryogenic temperature.

We will further fabricate the miniaturized RF receiver front-end by combining the small antenna with RF semiconductor devices described in [9], [10].

\section{REFERENCES}

[1] R. Azadegan and K. Sarabandi, "A novel approach for miniaturization of slot antennas," IEEE Trans. Antennas Propagat., vol. 51, no. 3, pp. 421-429, Mar. 2003.

[2] S. C. Gao, L. W. Li, M. S. Leong, and T. S. Yeo, "Dual-polarized slotcoupled planar antenna with wide bandwidth," IEEE Trans. Antennas Propagat., vol. 51, no. 3, pp. 421-429, Mar. 2003.
[3] K. Li, C. H. Cheng, K. F. Tong, T. Matsui, and M. Izutsu, "Millimeterwave coplanar patch and array antennas," in Proc. APMC 2002, Nov. 2002, pp. 833-836.

[4] A. Grbic and G. V. Eleftheriades, "Leakly CPW-based slot antenna arrays for millimeter-wave applications," IEEE Trans. Antennas Propagat., vol. 50, no. 11, pp. 1494-1504, Nov. 2002.

[5] M. S. A. Salameh, Y. M. M. Antar, and G. Seguin, "Coplanar-waveguide-fed slot-coupled rectangular dielectric resonator antenna," IEEE Trans. Antennas Propagat., vol. 50, no. 10, pp. 1415-1419, Oct. 2002.

[6] K. Satoh, T. Mimura, S. Narahashi, and T. Nojima, "Today and tomorrow of HTS technology applications," in MWE Microwave Workshop Digest, 2000, pp. 102-117.

[7] S. Ohshima, "High-temperature superconducting passive microwave devices, filters and antennas," Supercond. Sci. Technol., vol. 13, pp. 103-108, Jan. 2000.

[8] K. Yoshida, T. Takahashi, H. Kanaya, T. Uchiyama, and Z. Wang, "Superconducting slot antenna with broadband impedance matching circuit," IEEE Trans. Appl. Supercond., vol. 11, no. 1, pp. 103-106, Mar. 2001.

[9] H. Kanaya, Y. Koga, J. Fujiyama, G. Urakawa, and K. Yoshida, "Design and performance of high $T_{c}$ superconducting coplanar waveguide matching circuit for RF-CMOS LNA," IEICE Trans. Electron., vol. E86-C, no. 1, pp. 37-41, Jan. 2003.

[10] H. Kanaya, Y. Koga, G. Urakawa, and K. Yoshida, "Design of HTS coplanar waveguide matching circuit for low noise CMOS-HTS receiver," IEEE Trans. Appl. Supercond., vol. 13, no. 2, pp. 1031-1034, Jun. 2003.

[11] H. Kanaya, G. Urakawa, Y. Tsutsumi, T. Nakamura, and K. Yoshida, "High temperature superconducting slot array antenna connected with low noise amplifier," in Proc. Eucas 2003.

[12] G. L. Matthaei, L. Young, and E. M. T. Jones, Microwave Filters, Impedance-Matching Networks, and Coupling Structures. Norwood, MA: Artech House, 1980. 